\title{
Prediction of Short-term Evolution of Cloud Formations Based on Meteosat Image Sequences
}

\author{
Raffaele Bolla, Mario Marchese, Carlo Nobile, Sandro Zappatore \\ Department of Communications, Computer and Systems Science (DIST) \\ University of Genoa \\ Via Opera Pia, 13 - 16145 Genova, Italy
}

\begin{abstract}
In this paper an algorithm to predict the short-term evolution of cloud formations with high rainfall probability by using image sequences coming from meteorological satellites (Meteosat images) is described. The proposed algorithm consists of four steps: the first step performs image processing procedures (thresholding and relaxation, edge following, frequency filtering) adapted to a specific environmental application; the second step is dedicated to solve the correspondence problem in different images while the third one deals with the problem of modelling the parameters defining the time evolution of a cloud formation and investigates how these parameters can be estimated. The last step concerns the motion prediction of processed clouds. The main goals of this work are to test the effectiveness of the proposed procedure with different values of the filtering parameters, and to verify the importance and the influence of the mentioned parameters in the prediction mechanism. Some experimental results obtained by using real image sequences coming from Meteosat satellites are shown.
\end{abstract}

\section{Introduction}

The framework of this work is the processing of sequences of Meteosat images in order to extract information useful in a decision support system. The goal is to describe the evolution of cloud formations to foresee the rainfall probability in limited areas [1]. Specifically, an algorithm is presented to estimate the evolution parameters of cloud formations characterized by high rainfall probability in a Meteosat sequence. The input to the procedure is represented by the regions of interest, extracted in two or more consecutive images by means of processing steps applied to the infrared data. The proposed technique consists of four steps, as already described in [2]. In the first one specific image processing procedures are applied to the original images in order to extract the regions of interest in each image. Three different types of procedures are used: a first, thresholding and relaxation procedure and an edge follower are applied, then the obtained edges are filtered to detect their relevant features, thus improving the robustness of the following steps. The second step deals with the correspondence problem between regions of interest in different images. The correspondences are therefore determined by minimizing a cost function taking into account the distance among possible regions, as well as their difference in area. In the third step, the parameters defining the time evolution of a cloud formation are estimated. Such parameters are chosen to be those of a linear model, namely a translation vector, a rotation matrix and a deformation matrix. They are estimated by means of an iterative procedure that minimizes the not overlapping surfaces of two corresponding cloud formations. The last step consists of using data obtained from a sequence of images to predict the evolution parameters, i.e. the centre of mass coordinates and the deformation matrix, which are used to compute the rainfall regions of the next two images in the sequence. This task is performed by employing interpolating functions, written as a linear combination of orthogonal functions. The next Section describes the thresholding and relaxation procedures, and 
the clustering and filtering operations on the available image. In Section 3 the strategy to obtain the corresponding clouds in two consecutive images is described, while the approach to estimate the motion parameters is shown in Section 4. Section 5 presents some experimental results obtained from Meteosat imagery.

\section{Meteosat Image Processing}

The first step of the image processing concerns thresholding and relaxation. The goal of this step is to extract the regions characterized by high rainfall probability from a sequence of Meteosat images in the infrared band. According with Griffith and Woodley heuristics, the regions whose temperature is below a certain threshold are called clouds. In this way, the so called clouds are associated with a certain rain intensity $[3,4]$. In order to improve the robustness of the thresholding procedure, a technique, based on a relaxation method, has been applied to obtain a binarized image from the original grey scale IR image. The relaxation is, basically, a stochastic process $[5,6]$ that allows to classify image pixels into two distinct classes (cloud points and background) by taking into account not only the pixel value but also the characteristics of the neighbouring pixels. The aim of the procedure is to correct or to reduce the inherent errors involved in classifying a point according only to its properties. Thus the final binarized image results from removing the isolated cloud points surrounded by background points and from filling the small background areas inside a large set of cloud points. Cloud points identified by the relaxation procedure need to be clustered into some connected regions (called clouds in the following). This operation is performed by using a simple scheme based on the Sobel edge follower algorithm [7]. A blob is classified as a cloud if $90 \%$ of inner points is evaluated as belonging to the cloud point set. The last operation of the image processing step is the filtering, aimed at smoothing the contours (by means of lowpass operator in the frequency domain) obtained during the clustering; in fact, a too detailed information about the contours is not necessary for our goal and it could introduce a high computational burden. The filtering employs the Discrete Fourier Transform (DFT) whose mathematical details are described in the following: let us suppose to have $\mathrm{N}$ samples of a discrete signal $x(n)$ with $n=0 . . . N-1$; the $k$-th value $X(k)$ of the DFT of the signal $x(n)$ is

$$
X(k)=\sum_{n=0}^{N-1} x(n) e^{-j 2 \pi k n / N} \quad k=0 . . . N-1
$$

In this context the signal to be filtered is the cloud boundary; if the number of values is not a power of 2 (as it has to be to implement a frequency filter), the boundary is oversampled thus obtaining a correct number of samples (let $\mathbf{N}$ be this number). A generalized raised cosine function, $F(k)$ in (2), is used to cut-off all the frequencies higher than a fixed threshold $\mathrm{f}_{\mathrm{c}}$.

$$
F(k)=\left\{\frac{1}{2}\left[1-\sin \left[\frac{\pi \beta}{N \alpha}\left(k-\frac{N}{2 \beta}\right)\right]\right]\right.
$$

$$
\begin{gathered}
0 \leq \mathrm{k} \leq \mathrm{N}(1-\alpha) / 2 \beta \\
\frac{\mathrm{N}}{2 \beta}(1-\alpha)<\mathrm{k}<\frac{\mathrm{N}}{2 \beta}(1+\alpha) \\
\mathrm{k}>\mathrm{N}(1+\alpha) / 2 \beta
\end{gathered}
$$

Then by back-transforming the filtered values of the boundary in the space domain are obtained. The filter allows to choose precisely the cut-off frequency and the smoothing level of the filter. The effect of the frequency filter and his impact on the prediction is extensively described in Section 5. 


\section{Cloud Identification}

After the filtering, a crucial step is the identification of corresponding clouds in two consecutive images. A cloud in two consecutive frames can change position, orientation, scale; moreover, it can split, or merge other clouds generating different cloud formations. More specifically, because of winds and atmospheric currents a cloud can split into two or more parts, thus creating other clouds not existing before; on the other hand, two or more clouds can merge giving birth to one larger cloud. It can be noted that an automatic procedure detecting splitted and merged clouds is very difficult to derive. In our approach the identification is performed in three steps: case of no splitting and merging, case of splitting, case of merging. In the first case the operation is performed by setting a variable side rectangle around the center of mass of a cloud in the first image, then by searching if some centers of mass in the second image fall inside the given rectangle; if more than one center of mass satisfies this condition a cost function is evaluated to choose the 'best' corresponding cloud. The cost function mentioned above is based on: i) the distance between centers of mass, ii) the difference of areas. That is:

$$
\operatorname{cost}=B * \sqrt{\left(x_{b, j}-x_{b, i}\right)^{2}+\left(y_{b, j}-y_{b, i}\right)^{2}}+A * \mid A_{\text {rea }}-\text { Area }_{i} \mid
$$

Being

$x_{b, i}, x_{b, j}$, the $x$-values of the considered centers of mass in the first and second $y_{b, i}, y_{b, j}$ the $y$-values of the considered centers of mass in the first and second Area $_{i}$, Area ${ }_{j}$ the area of the considered clouds in the first and second image,

The mechanism is repeated for every cloud inside the first image; if the first step does not identify all the clouds, the case of possible splitting is investigated for not identified clouds. The operation is performed by setting a rectangle around each cloud in the first image; it is important to note that the mentioned rectangle is not set around the center of mass but it contains every point of the cloud. The second operation step is performed by examining if some centers of mass in the second image are inside the rectangle, then every possible combination for all found centers of mass is evaluated and the corresponding cloud is chosen by computing the mentioned cost function for every permutation. After finding the splitted clouds, the last operation is the evaluation of merged blobs. This step is performed by the same mechanism as in the previous case; the difference is that the rectangle is set on the second image and not on the first one.

\section{Motion Modelling and Prediction}

Before predicting the future position of a cloud it is necessary to model the cloud motion itself. For the sake of simplicity the motion model employed in our approach is linear, meaning that the movement of each point $x_{i}=\left(x_{i}, y_{j}\right)$ of the cloud can be
described $[8,9]$ as:

$$
\underline{x}_{i}=\underline{d}+F_{\underline{x}-1}
$$

Being: $\underline{x}_{i-1}$ the position of the point at instant $i-1, \underline{x}_{i}$ the position of the point at instant $i, \underline{d}$ the translation vector, $F$ the shape matrix. By using the Polar Decomposition Cauchy theorem the shape matrix F can be written as the product of two matrices $\mathrm{R}$ and $\mathrm{U}$ : 


$$
\mathrm{F}=\mathrm{RU}
$$

with $\mathrm{R} \in \mathrm{O}^{+}$(positive definite) and $\mathrm{U} \in \mathrm{Sym}$ (Symmetric matrixes). From a physical point of view, the matrix $R$ can be considered the rotation matrix describing the cloud rotation of an angle $\theta$ around an axis through the centre of mass and orthogonal to the plane of the cloud pattern:

$$
R=\left[\begin{array}{cc}
\cos \theta & \sin \theta \\
-\sin \theta & \cos \theta
\end{array}\right]
$$

The matrix $U$ can be interpreted as the matrix containing the information about the deformation of the cloud:

$$
U=\left[\begin{array}{ll}
u_{11} & u_{12} \\
u_{21} & u_{22}
\end{array}\right] \text { with } u_{12}=u_{21}
$$

The problem is then reduced to the evaluation of the translation vector $\mathbb{d}$ and the matrix $F=R U$. The vector $\mathbb{d}$ is computed as the difference of the cloud centers of mass in two consecutive frames, the matrix $F$ is evaluated by a minimization algorithm based on not overlapped surfaces. The estimated parameters associated with the motion and the deformation of a cloud during the transitions between successive frames can be exploited for an efficient prediction of the position and the shape of the cloud in the next unknown frame. The estimation of the center of mass parameters $\left(\mathrm{x}_{\mathrm{b}}, \mathrm{y}_{\mathrm{b}}\right)$, the rotation angle $\theta$ and the deformation matrix $\mathrm{U}=\left\{\mathrm{u}_{11}, \mathrm{u}_{12}, \mathrm{u}_{21}=\mathrm{u}_{12}, \mathrm{u}_{22}\right\}$ are obtained by using an interpolating function and having as available data: 1) $\mathbf{M}$ previous images in successive instants $\left.\left(\mathrm{t}_{0}, \ldots \mathrm{t}_{M-1}\right), 2\right) \mathrm{M}$ values of $\left(\mathrm{x}_{b}, \mathrm{y}_{b}\right)$ for each blob, 3) M-1 values of $\theta$ for each blob, 4) M-1 values of $U$ for each blob.

The succession given by the component of the cloud center of mass is interpolated by a suitable function. Once we have the analytical expression of this function we are able to determine the next coordinates of the center of mass by simple substitution of the desired instant. The used strategy involves the use of orthogonal functions; for a detailed description of the interpolation method see [10].

\section{Experimental Results}

In this Section some experimental results are shown, that confirm the effectiveness of the presented prediction strategy. The prediction mechanism has been applied to two sequences of three consecutive images obtained from the same original sequence by using two different cut-off frequencies in the filtering procedure. The sequences contain just a cloud because the aim is to test the prediction mechanism and the presence of other clouds would have made the analysis of the results more difficult. Fig.1 shows the sequence obtained by using the value $\beta=4$ in the filter. Fig. 2 and Fig. 3 show, respectively, the next predicted image (one step forward prediction), and the image predicted after the next (two steps forward prediction) along with the cloud shape really observed from the METEOSAT images. Results shown in Fig.2 and Fig. 3 have been obtained by using the image sequence in Fig.1 as an input. It can be seen that the predicted and the observed shapes are not quite different; little differences can be noted but they derive from the linear model used to describe the motion and deformation of a cloud. It is important to note that the similarity of the shapes is just a qualitative measure of the quality; taking into account the used motion model, for a quantitative evaluation, the analysis of the position of the center of mass and of the rotation angle (reported in the following) are more meaningful. Fig. 4 is the image sequence obtained by using the value $\beta=128$ in the filter, that implies a lower cut-off frequency; this sequence is used as an input to 
get Fig.5 and Fig. 6 which have the same meaning as Fig. .2 and Fig. 3. It can be seen that these last images contain a less detailed information then the previous; however ignored details could not be predicted (as can be seen in Fig. 2 and 3) by using the proposed modelling scheme.

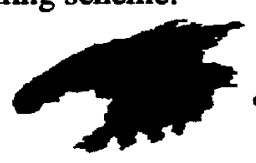

Image 0

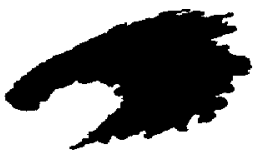

Image 1

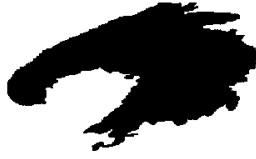

Image 2

Fig.1. Blobs used in the prediction.

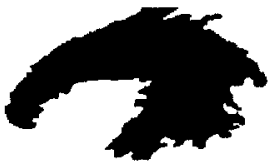

Predicted blob

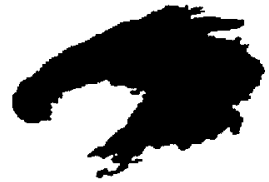

Observed blob

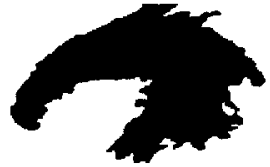

Predicted blob

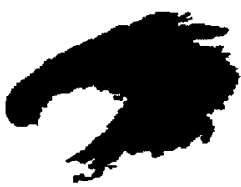

Observed blob

Fig.3. Two step predicted blob and observed blob of the image 4 .

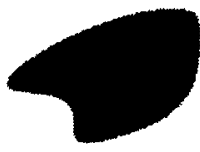

Image 0

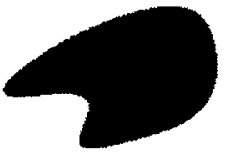

Image 1

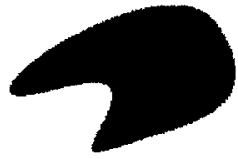

Image 2

Fig. 4. Blobs used in the prediction.

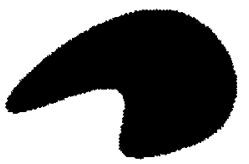

Predicted blob

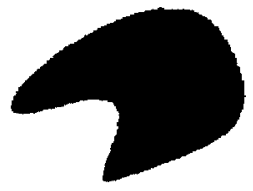

Observed blob

Fig. 5. The one step predicted blob and observed blob of the image 3 .

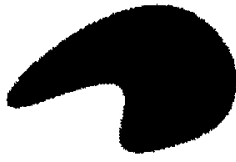

Predicted blob

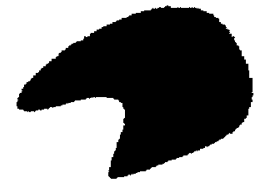

Observed blob observed blob of the image 4 .

In Fig. 7 the behaviour of the observed rotation angle and of the predicted one for images 3 and 4 is depicted versus time; it can be noted that the predicted value is close the observed one, especially in the one step prediction. The same consideration can be made for the behaviour of the center of mass coordinates depicted in Fig. 8 . The results shown concern only one situation but they are representative of the average behaviour of the described prediction mechanism.

\section{Conclusions}

In this paper an algorithm to estimate the evolution parameters of cloud formations characterised by high rainfall probability in a Meteosat sequence has been presented. This algorithm consists of four steps: a first step, described in Section 2, of specific image processing procedures, namely a thresholding, a relaxation, an edge following and a filtering procedure, used to detect the clouds and their relevant features; a second step, described in Section 3, dealing with the correspondence problem; a third step in which the parameters defining the time evolution of a cloud 
formation are estimated by using a linear model and, finally, the last step in which the prediction of the evolution parameters, i.e. the centre of mass coordinates and the deformation matrix, is computed, as described in Section 4. The aim of this technique was to predict the one or two step forward evolution of the clouds by using a sequence of three or four Meteosat images. The experimental results, presented in Section 5, show that this kind of algorithm achieves this goal by providing an acceptable prediction of the first and the second forward step evolution parameter.

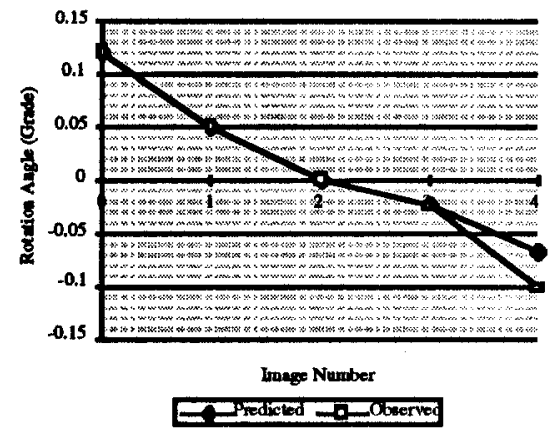

Fig. 7. Behaviour of the rotation angle values and predicted angle values for image 3 and 4 versus time with $\beta=4$.

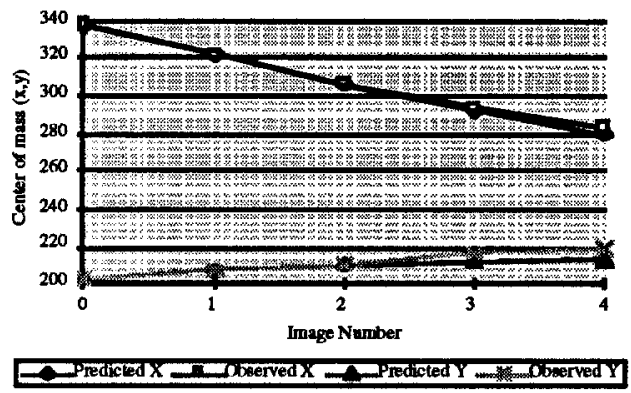

Fig.8. Behaviour of the center of mass vs time with $\beta=4$.

\section{References}

1. C.Braccini, G.Gambardella, A.Grattarola, S.Zappatore, "Meteosat image processing for clouds analysis and tracking", Proc. Workshop on "The role of radar in the Arno Project", Florence, Nov. 20-23, 1990.

2. R.Bolla, M.Marchese, C.Nobile, S.Zappatore "Estimating cloud formation evolution from sequences of METEOSAT images", Proc. 2nd ACM Workshop on Adv. in Geo. Inf. Systems, Gaithersburg, MD, Dec. 1994.

3. R.F.Adeler, A.J.Negri, P.J.Wetzel, Rain Estimation from Sattelite: "An Examination of the Griffith-Woodly Technique", J. of Climate and Applied Meteorology, Vol.23,1983

4. G.C.Griffith, P.G.Grube, D.W.Martin, J.E.Stoud, D.N.Woodley, Rain Estimation from Geosynchronous Satellite Imagery- visible and Infrared Studies, Mon. Weather Rev. 106,1978

5. A.Touzani, J.G.Postaire "Mode detection by Relaxation" IEEE trans. on Pattern Anal. and Machine Intell. Vol. P.A.M.I. 10, N 6, 970-978, November 1988.

6. A.Rosenfeld and Russel C.Smith "Thresholding using Relaxation" IEEE trans. on Pattern Anal. and Machine Intell. Vol. P.A.M.I. 3, N ${ }^{\circ}$ 5, 588-606, Sept. 1981.

7. I.Sobel, "Neighborhood coding of binary images for fast contour following and general binary array processing", Comp. Graph. Im. Proc., vol.8, 1978, pp.127135.

8. S.Chadhuri, S.Chatterjee, "Motion analysis of a homogeneously deformable object using subset correspondences", Pattern Rec., vol.24, No. 8,1991,pp.739745.

9. D.Skea at al.,"A control point matching algorithm", Pattern Recognition, vol.26, No. 2, 1993, pp. 269-276.

10. H.F.Harmuth,"Transmission of information by orthogonal functions", SpringerVerlag, 1969, New York, USA. 